41. The extraction instrument must rest only on the tooth to be extracted without any impingement on the soft tissues and avoid any force on adjacent teeth or restorations

- Avoid excessive apical force on maxillary posterior teeth in close proximity to the antral floor and mandibular molars in close proximity to the inferior alveolar nerve

- Always use a controlled force and see what the tooth wants to do. If the tooth is stubborn and no movement is achieved, revaluate the radiographs to rule out anatomical variations (dilaceration, hypercementosis, ankylosis, accessory roots etc). Consider a surgical approach if within the competency of the operating dentist; otherwise, it may be best to stop the procedure and consider a specialist referral.

\section{Provide good post-extraction management}

Several measures are required to achieve uneventful healing following a tooth extraction: ${ }^{5}$

- After a successful tooth extraction, avoid the temptation to place a bite pack within the next microsecond. Palpate the socket walls to rule out the presence of any sharp bone and smooth with a bone file or rose-head surgical bur if required. Look carefully down the socket and use saline irrigation and suction to clear away any debris in the socket and reduce the microbial load. These measures are important to ensure uncomplicated healing of the extraction socket

- Achieve haemostasis prior to discharging the patient and never send the patient home with a bite pack in situ. A pressure pack placed directly over the extraction socket and held securely with the bite for ten minutes is usually adequate to achieve haemostasis.
Patients must avoid talking to the dentist/nurse during this time as it may compromise haemostasis. Patients on blood thinners may require additional local measures to achieve haemostasis ${ }^{1}$

- Provide written and verbal post extraction instructions re pain control, postoperative bleeding, oral hygiene, diet and things to avoid (smoking, alcohol, heavy exercise). Patients must be instructed to avoid active spitting/rinsing for at least 24 hours after extractions as it may dislodge the blood clot in the socket, compromise healing and possibly risking the development of alveolar osteitis

- Provide an emergency contact number and arrange a follow-up for complicated extractions.

Finally, dentists may consider additional training to develop their extraction skills specially to master safe and effective use of luxators and elevators and to carry out surgical tooth extractions. It may be worth exploring opportunities to work some sessions at local oral surgery units in primary and secondary care or local dental schools. Given the opportunity, such exposure may provide invaluable experience to dentists interested in improving their oral surgery skills.

\section{References}

1. Toole J, McKenna G, Smyth J. Managing patients at risk of medication related complications requiring dental extractions in primary care. Prim Dent J 2020; 9: 54-58

2. Wilmott $S$, Yates J, Pretty I A. Dental extractions in primary care for patients at risk of MRONJ. Br Dent J 2021; doi: 10.1038/s41415-021-3674-8.

3. Hupp M R, Ellis J R, Tucker E. Contemporary oral and maxillofacial surgery. 6th edition. pp 92-112. Missouri: Elsevier, 2008.

4. Dargue A, Fyfe E, French K et al. The impact of wrong-site surgery on dental undergraduate teaching: a survey of UK dental schools. Eur J Dent Educ 2021; 25: 670-678.

5. Sambrook P J, Goss A N. Contemporary exodontia. Aust Dent J 2018; DOI: 10.1111/adj.12586

\title{
Government dragging heels on overseas dentists left unable to practise
}

The British Dental Association (BDA) has called on Ministers and the General Dental Council (GDC) to deliver rapid change to laws governing tests for overseas dentists, after COVID restrictions have left thousands of applicants waiting to pass registration exams to practise in the UK.

The BDA has warned that a three-month consultation on necessary legal changes - launched on 7 February - will mean little chance of parliamentary time until the autumn at the earliest, leaving an incredibly narrow window until mutual recognition of qualifications for EU dentists ends in December.

Due to the pandemic, the Overseas Registration Exam (ORE) has not taken place since January 2020. In response to a parliamentary question, Minister Maria Caulfield MP revealed that there are over 2,000 applicants currently waiting for an opportunity to sit these exams. Under existing arrangements, most of these dentists will end up waiting years before being allowed a chance to practise in the UK and due to outdated regulations, many won't be allowed to work in the NHS.

The GDC recently told 132 candidates who had already passed the first part of their ORE that they cannot currently be allocated a place to sit Part 2 as - due to no exams taking place for two years - they have exceeded the permitted waiting time of five years from the first attempt at Part 1.

In messages to these candidates on 3 December 2021, the GDC said there was nothing it could do given the legislation it works to. The consultation that has now been published addresses the imminent issues for these candidates but the timescales involved mean further delays to meaningful change. The consultation had been expected since the middle of 2021 and it is not clear why it has taken so long to get off the ground. The BDA will consider all proposals and provide relevant feedback but stressed government must act quickly on feedback.

The BDA has been overwhelmed by messages from candidates citing desperation, suicidal thoughts and immense anger at the current situation. It has heard from dentists who have postponed having children because of the delays and who have had to work in other roles such as minicab drivers, security guards, busboys and supermarket shelf stackers to make ends meet.

The BDA had pressed both the GDC and Health Secretary, Sajid Javid to work at pace to resolve the situation and bring down the backlog awaiting examinations and improve the processes which allow overseas dentists to work in the NHS.

Patients in England face profound access problems, with over 38 million NHS appointments lost since the first lockdown and with 
1 nearly 1,000 dentists leaving NHS services in the last year.

BDA Chair Eddie Crouch said: 'every new colleague unable to sit this exam translates into thousands of patients that will be denied access to needed care.

'Inaction has left highly skilled clinicians waiting tables and stacking shelves while millions struggle to get an NHS appointment.

'For the sake of our patients, government must ensure that these dentists are supported and those still waiting to sit the exams can look forward to a fairer system?

\section{Notice of death}

David Boswell studied dentistry at Guy's Hospital from 1945 and practised as a dentist from 1951 in the Bath/Midsomer Norton area. David died at home in Timsbury surrounded by his family on 1 February 2022. Loving husband, father, grandfather and great-grandfather.

His funeral service was held in Timsbury, near Bath, earlier in February. Donations to Dorothy House and Smile Train.

\section{Research to examine the role dentists can play in tackling obesity crisis}

A new study being led by Loughborough University will look at the role dentists can play in reducing obesity in the UK.

Over $50 \%$ of the global population are living with being overweight and are at increased risk of developing diseases such as type 2 diabetes, heart disease and cancer.

GPs and practice nurses have for some time used their patient consultations to raise the subject of weight management. But despite dentists already delivering behaviour change messages for oral health during their consultations - for example smoking cessation and reducing the consumption of sugar sweetened beverages - the role they can play in tackling obesity has so far been overlooked.

Now researchers from Loughborough will be working directly with dentists to assess how they can provide weight management interventions to their patients.

It will look at what can be learnt from the NHS Making Every Contact Count campaign, providing training for dentists on how to have constructive conversations about weight management with their patients.

The study is being led by Amanda Daley, a Professor of Behavioural Medicine based in the university's Centre for Lifestyle Medicine and Behaviour (CLiMB).

Professor Daley said: 'the case for dentists to be involved in reducing population obesity is strong. Like GPs, dentists are part of primary care health services and are ideally positioned to screen and intervene to reduce obesity.

'They typically consult with most of the population at least yearly, meaning they could routinely embed efficient monitoring of weight into dental health services and offer interventions at scale to both adults and children.

'If we are serious about reducing obesity in the population, it will require an "all hands-on deck" approach, including active advocacy from dental health professionals.'

Jessica Large, a dentist working with the project team, said: 'it is important that we look at how all health professionals are able to contribute to reducing obesity in the public and dentists might be able to help in a positive way to achieve this.

'Routine body mass index screening for children and healthy weight discussions are already ongoing in some hospital dental

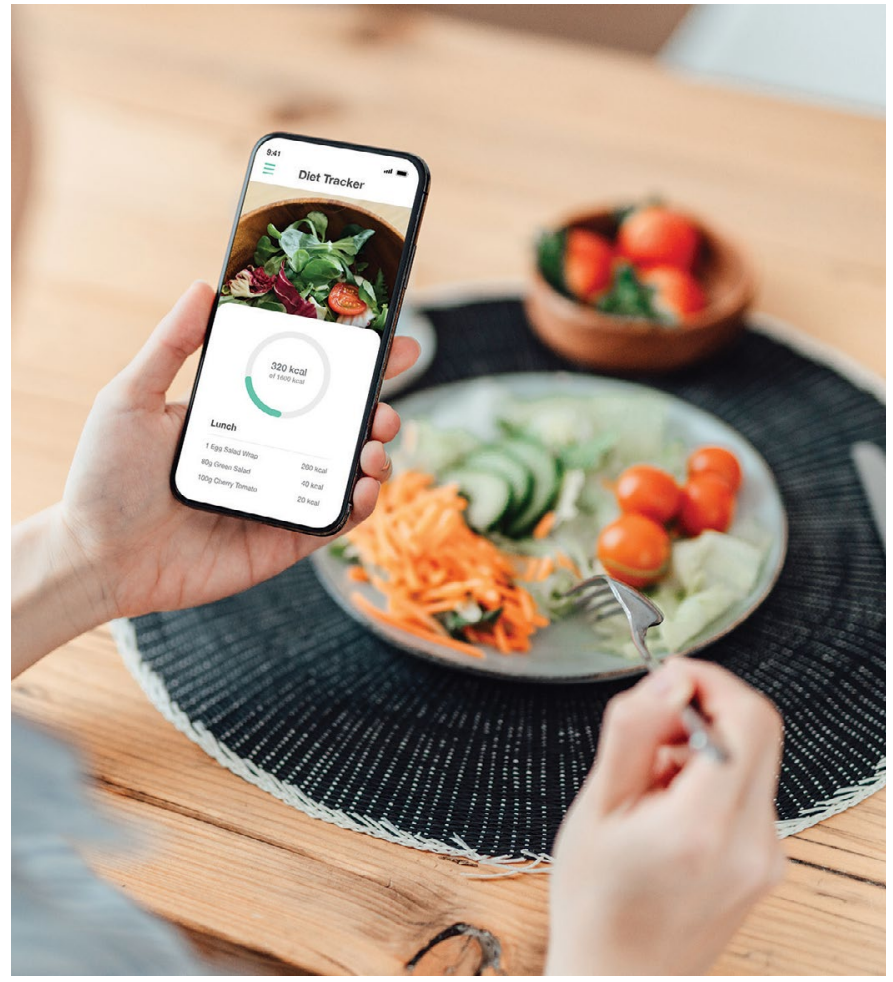

settings, with positive feedback from families and dental teams. I am looking forward to exploring the wider acceptability amongst the profession and public.'

The research is being funded via the National Institute for Health Research Research Professorship programme and will run for two years. Any dentists interested in being part of the project should contact the CLiMB team at climb@lboro.ac.uk.

Professor Daley has published an opinion piece on the role dentists have to play in tackling obesity in the BDJ: 'Time to get our teeth into reducing obesity: should dentists screen and deliver interventions to reduce obesity in the population? ${ }^{1}$

\section{Reference}

1. Daley A J. Time to get our teeth into reducing obesity: should dentists screen and deliver interventions to reduce obesity in the population? Br Dent J 2022; 232: 78-79. 